\title{
PARP Inhibitors may be Beneficial in a Broader Range of Patients
}

\author{
An Expert Interview with W Lee Kraus
}

Cecil H. and Ida Green Center for Reproductive Biology Sciences, Department of Obstetrics \& Gynecology, University of Texas Southwestern Medical Center, Dallas, TX, USA

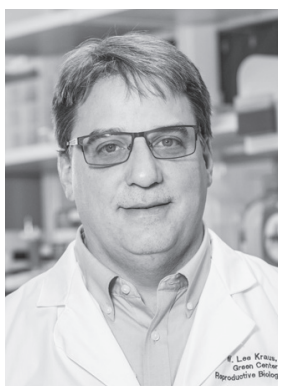

\section{W Lee Kraus}

W Lee Kraus, PhD is Professor and Director of the Cecil H. and Ida Green Center for Reproductive Biology Sciences, Vice Chair for Basic sciences in the Department of Obstetrics \& Gynecology, Assistant Director for Basic Science in the Simmons Comprehensive Cancer Center, and Professor of Obstetrics \& Gynecology and Pharmacology at University of Texas Southwestern Medical Center in Dallas. Dr Kraus received a BS in Animal Physiology from Cornell University in 1989 and a PhD in Physiology and Molecular Biology from the University of Illinois, UrbanaChampaign in 1994. He completed his postdoctoral research on the mechanisms of transcriptional regulation at the University of California, San Diego in 1999. From 1999-2010, Dr Kraus was a professor in the Department of Molecular Biology and Genetics at Cornell University in Ithaca, New York, with a joint appointment in the Department of Pharmacology at the Weill Medical College of Cornell University in New York City. In July 2010, Dr Kraus moved to UT Southwestern Medical Center in Dallas where he holds the Cecil H. and Ida Green Distinguished Chair in Reproductive Biology Sciences. He has received numerous awards, including a Burroughs Wellcome Fund Career Award in the Biomedical Sciences, the Cornell University College of Agriculture and Life Sciences Young Faculty Teaching Excellence Award, and the Richard E Weitzman Memorial Award and the Ernst Oppenheimer Award from the Endocrine Society.

\section{Keywords}

BRCA1/2, PARP inhibitors, breast cancer

Disclosure: W Lee Kraus is a founder, consultant, and SAB member for Ribon Therapeutics, Inc. He is also a co-holder of U.S. Patent 9,599,606 covering a set of ADP-ribose detection reagents, which have been licensed to and are sold by EMD Millipore.

Acknowledgment: Medical writing assistance was provided by Katrina Mountfort of Touch Medical Media, and supported by Touch Medical Media.

Review Process: This is an expert interview and, as such, has not undergone the journal's standard peer review process

Compliance with Ethics: This article involves a review of literature and does not report on new clinical data, or any studies with human or anima subjects performed by any of the authors.

Authorship: The named author meets the Internationa Committee of Medical Journal Editors (ICMJE) criteria for authorship of this manuscript, takes responsibility for the integrity of the work as a whole, and has given final approval to the version to be published.

Received: August 14, 2019

Accepted: October 1, 2019

Citation: Oncology \& Hematology Review (US). 2019; 15(2):66-7

Corresponding Author: W Lee Kraus, Cecil H. and Ida Green Center for Reproductive Biology Sciences, The University of Texas Southwestern Medical Center at Dallas, 5323 Harry Hines Boulevard, Dallas, TX 75390-8511, USA. E: LEE.KRAUS@utsouthwestern.edu

Support: No funding was received in

the publication of this article.
$\mathrm{O}$ laparib was the first PARP inhibitor to be approved by the US Food and Drug Administration (FDA) in 2014 for the treatment of ovarian cancer containing BRCA1/2 mutations. ${ }^{1}$ Since then niraparib and rucaparib have been approved for the treatment of ovarian cancer and in 2018 olaparib and talazoparib received FDA approval for use in patients with germline BRCA-mutated, human epidermal growth factor receptor 2 (HER2)-negative, advanced breast cancer previously treated with chemotherapy and/or endocrine therapy. ${ }^{2}$ However, the prevalence of BRCA mutations is low and, as a consequence, PARP inhibitors are currently used to treat less than $10 \%$ of patients with breast cancer. ${ }^{3,4}$

Recent research has found that PARP inhibitors can be effective in patients without a BRCA mutation, since they also act through an alternative molecular pathway. In an expert interview, Dr Kraus describes this pathway and provides insights into the potential broader therapeutic applications of PARP inhibitors.

\section{Q. What is the role of PARP-1 in the pathogenesis of cancer?}

PARP-1 is a protein that resides in the cell nucleus where it plays a role in most, if not all, nuclear functions (e.g. DNA repair, transcription, RNA processing, RNA ribosomal transcription). PARP-1 uses these pathways to assist the cancer cell in its uncontrolled growth in a number of ways. These include promoting alternate or enhanced activities in these pathways, or perhaps reducing cell growth control through mutation or functional inactivation of PARP-1 or other key proteins. Essentially, it helps the cancer cell to harness normal pathways that exist in the cell for a pathological growth advantage.

\section{Q. What is known about the mechanism of action in of PARP inhibitors in BRCA1/2-mutant cells?}

The BRCA1/2 proteins play a variety of roles in important DNA repair functions, including checkpoint activation, DNA replication, and DNA repair involving homologous recombination. When the genes encoding those proteins are mutated, it causes a variety of defects that impair the ability of the cell to maintain the integrity of its genome. Initially, defects in the pathways controlled by the BRCA1/2 proteins can cause a growth advantage for the cell. If DNA replication and repair and the checkpoint pathways are not functioning properly, this may cause mutations in the genome and allow the cell to divide without repairing those mutations, causing a growth advantage. The PARP inhibitors promote a therapeutic condition known as synthetic lethality in BRCA1/2-mutated cells, meaning that there are 
now two defects, one that comes from a genetic lesion and one that comes from the drug. Even the cancer cell is unable to overcome the genetic damage and the DNA replication stress caused by the combination of the drug and the genetic lesion, preventing the cancer cell from being able to grow and ultimately promoting the death of the cell.

\section{Q. Could you describe the alternative pathway that has been identified in BRCA1/2-intact breast cancer cells?}

This alternative pathway, which has been identified in breast cancer cells that are intact for BRCA1/2, functions independently of DNA repair. This is a pathway that involves the production of ribosomal RNA, a special type of non-coding RNA that is critical for assembling ribosomes, which are used translate proteins from mRNAs in the cytosol of the cell. Protein translation is critical for the growth and proliferation of the breast cancer cell. The cancer cells are addicted to making as many proteins as possible so they can proliferate aggressively. PARP-1 plays a critical molecular role in ribosomal RNA production, which occurs in a specialized compartment in the cell nucleus called the nucleolus. Through its catalytic activity, which can be inhibited by PARP inhibitors, PARP-1 modifies a protein called DDX21, which is critical for ribosomal RNA transcription. Inhibiting this pathway reduces cellular ribosome content, which slows the growth of the cancer cells.

\section{Q. Are there any particular tumor types that are likely to respond to inhibition of this pathway?}

We have found that the localization of DDX21 in the nucleolus is a good indicator of the activity of this alternate pathway, therefore we think that DDX21 may be a good biomarker. We are now surveying a broad range of cancers to see if a shift in localization of DDX21 can serve as a marker of
PARP activity and possibly response to PARP inhibitors. Expanding this to additional cancer types beyond breast cancer is important for this research. We are looking at ovarian cancer, which was the first cancer for which PARP inhibitors were approved, and also prostate cancer and pancreatic cancer. Since this is a common pathway, it could play a role in response to PARP inhibitors in many other types of cancer.

\section{Q. What are the clinical implications of these findings?}

These findings open up a new category of cancers that may show a clinical benefit from the use of PARP inhibitors. At the moment, the FDA has only approved the PARP inhibitors in BRCA1/2 mutant cells. Because we have identified an alternative pathway that does not require DNA damage or BRCA mutations, we may find additional patients who might be good candidates for the use of PARP inhibitors. This fits well with growing evidence from basic and preclinical studies, and even some clinical studies, showing that there is broader efficacy of PARP inhibitors beyond BRCA1/2mutated cancers.

\section{Q. What studies have been performed to date and what further studies are planned?}

Much of the work done to date was to define the molecular mechanisms of this pathway. In addition, some studies in mouse xenograft models and patient samples helped to show that this pathway is active in human cancers. ${ }^{5}$ These have been exclusively in breast cancers and they clearly showed that PARP inhibitors were effective in BRCA1/2 intact breast cancers. They also showed that this pathway requires PARP-1 activity and that DDX21 plays a critical role in this pathway in human breast cancers. In the future, we will need prospective clinical studies to fully understand the clinical implications of these findings. $\square$

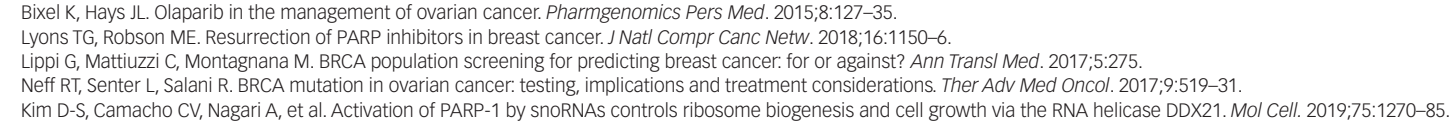

